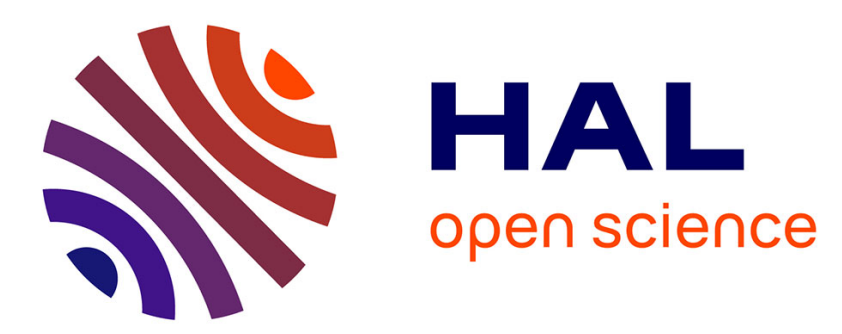

\title{
Overlay of Multicast Service in WDM-PON Based on Dynamic Wavelength Reflection Scheme
}

Min Zhu, Shilin Xiao, Wei Guo, He Chen, Anne Wei, Yaohui Jin, Weisheng $\mathrm{Hu}$, Benoit Geller

\section{- To cite this version:}

Min Zhu, Shilin Xiao, Wei Guo, He Chen, Anne Wei, et al.. Overlay of Multicast Service in WDMPON Based on Dynamic Wavelength Reflection Scheme. Communications and Photonics Conference and Exhibition (ACP), 2009, Shanghai, China. 10.1364/ACP.2009.TuF3 . hal-01225812

\section{HAL Id: hal-01225812 \\ https://hal.science/hal-01225812}

Submitted on 6 Nov 2015

HAL is a multi-disciplinary open access archive for the deposit and dissemination of scientific research documents, whether they are published or not. The documents may come from teaching and research institutions in France or abroad, or from public or private research centers.
L'archive ouverte pluridisciplinaire HAL, est destinée au dépôt et à la diffusion de documents scientifiques de niveau recherche, publiés ou non, émanant des établissements d'enseignement et de recherche français ou étrangers, des laboratoires publics ou privés. 


\title{
Overlay of Multicast Service in WDM-PON Based on Dynamic Wavelength Reflection Scheme
}

\author{
Min Zhu ${ }^{\mathrm{ab}}$, Shilin Xiao* ${ }^{\mathrm{a}}$, Wei Guo ${ }^{\mathrm{a}}$, He Chen ${ }^{\mathrm{a}}$, Anne Wei ${ }^{\mathrm{c}}$, \\ Yaohui Jin ${ }^{\mathrm{a}}$, Weisheng $\mathrm{Hu}^{\mathrm{a}}$ and Benoit Geller ${ }^{\mathrm{d}}$ \\ ${ }^{a}$ State Key Lab of Advanced Optical Communication Systems and Networks, Dept. of Electronic \\ Engineering, Shanghai Jiao Tong University, 800 Dongchuan Rd, Shanghai 200240, China; \\ ${ }^{\mathrm{b}}$ SATIE Lab, ENS de Cachan, 61, avenue du President Wilson, 94230, Cachan, France; \\ ${ }^{c}$ ATTIS Lab, Université de Toulouse II, 31703, Blagnac, France; \\ ${ }^{\mathrm{d}}$ UEI Lab, ENSTA ParisTech, 32, boulevard Victor, 75739, Paris, France; \\ *Corresponding author: slxiao@sjtu.edu.cn
}

\begin{abstract}
We propose a novel scheme to overlay multicast service over a wavelength-division-multiplexed (WDM) passive optical network (PON) based on dynamic wavelength reflection scheme. At the optical line terminal (OLT), for each WDM channel, a dynamic wavelength reflector is used to selectively bypass the corresponding optical carrier to avoid multicast data superimposition, while the remaining optical carriers go through the dynamic wavelength reflector and simultaneously carry the multicast data. The proposed scheme is experimentally demonstrated with 5-Gb/s downstream unicast, multicast data and $625-\mathrm{Mb} / \mathrm{s}$ upstream re-modulated data.
\end{abstract}

Keywords: WDM-PON, multicast, dynamic wavelength reflection, fiber bragg grating (FBG)

\section{INTRODUCTION}

WDM-PON is regarded as a promising solution for next generation broadband access networks because of its almost unlimited bandwidth, network security, simple management and upgradeability. To deploy multicast service over WDMPON, some efforts have already been reported ${ }^{1-5}$, and fall into three categories: 1) In-band transmission ${ }^{1,2}$, the multicast service shares the downstream wavelength bandwidth with unicast data service via time-division multiplexing scheme or orthogonal modulation format, which requires complicated timing or synchronization control; 2) Out-of-band transmission ${ }^{3,4}$, multicast service is overlaid on the same wavelength channel by means of sub-carrier multiplexing (SCM), which requires high-frequency electronic components at both the transmitter and receiver sides; 3) Out-ofwavelength transmission ${ }^{5}$, a additional specific wavelength channel is used to transmit the multicast service with simple optical power splitter and the arrayed waveguide grating (AWG). However, to accommodate the dynamic change of members in the multicast group, the dynamic deployment of broadcast/multicast service over WDM-PON needs to be further studied.

In this paper, a simple scheme to overlay multicast service is proposed. It uses a set of dynamic wavelength reflector added on each wavelength channel. When the dynamic wavelength reflector is in the bar (straight-through) state, the multicast data is orthogonal-overlaid with downstream unicast data on the corresponding optical carrier. The multicast data could not be superimposed when the dynamic wavelength reflector is in the reflection state to bypass the corresponding downstream optical carrier. By dynamically adjusting the state of the dynamic wavelength reflector, the multicast data can be enabled on a unicast wavelength channel to achieve selective multicast and thus realizing flexible network functions. We experimentally demonstrate the proposed WDM-PON with multicast overlay at 5-Gb/s and investigate its feasibility and system performance. The upstream data re-modulation based on the downstream nonreturn-to-zero (NRZ) format with a low extinction ratio (ER) is also demonstrated.

\section{PROPOSED SCHEME AND PRINCIPLE}

The proposed WDM-PON architecture with multicast overlay is shown in Fig. 1. In the optical line terminal (OLT), the downstream carrier of each wavelength channel is generated by a continuous-wave $(\mathrm{CW})$ laser, and modulated by an 
optical intensity modulators (IMs), to generate NRZ unicast data, with an extinction ratio (ER) of around $3 \mathrm{~dB}$. The lower ER makes it easier that the successful superimposition of the differential phase-shift keying (DPSK) multicast data and upstream data re-modulation ${ }^{5}$. To realize flexible multicast, the dynamic wavelength reflector can be added in between IM and arrayed waveguide grating (AWG) on each wavelength channel. In the schematic diagram as shown in the Fig. 1, the dynamic wavelength reflector consists of an optical circulator and a controllable Fiber Bragg Grating (FBG).

When an optical network unit (ONU) on a wavelength makes a request for the multicast service, the controllable FBG in the corresponding wavelength channel is adjusted via the control circuit to deviate from center wavelength and to allow unicast data carrier go straight to the AWG. Thus multicast signal can be simultaneously modulated on the downstream unicast carrier by an optical phase modulator (PM). To disable the multicast signal for an ONU, the FBG is dynamically tuned and reflects the corresponding wavelength to circulator and another AWG, so as to bypass multicast signal modulation. Hence the multicast signal can be selectively and dynamically superimposed onto downstream unicast signal on arbitrary wavelength channels. Then these two multiplexed signals I and II are combined by coupler and carried to remote node $(\mathrm{RN})$ and finally routed to individual ONU. It should be noted that all the multicast control intelligence is deployed at OLT by adjusting a set of the dynamic wavelength reflector (FBG and circulator), which is transparent to all ONUs and can be a cost-effective solution.

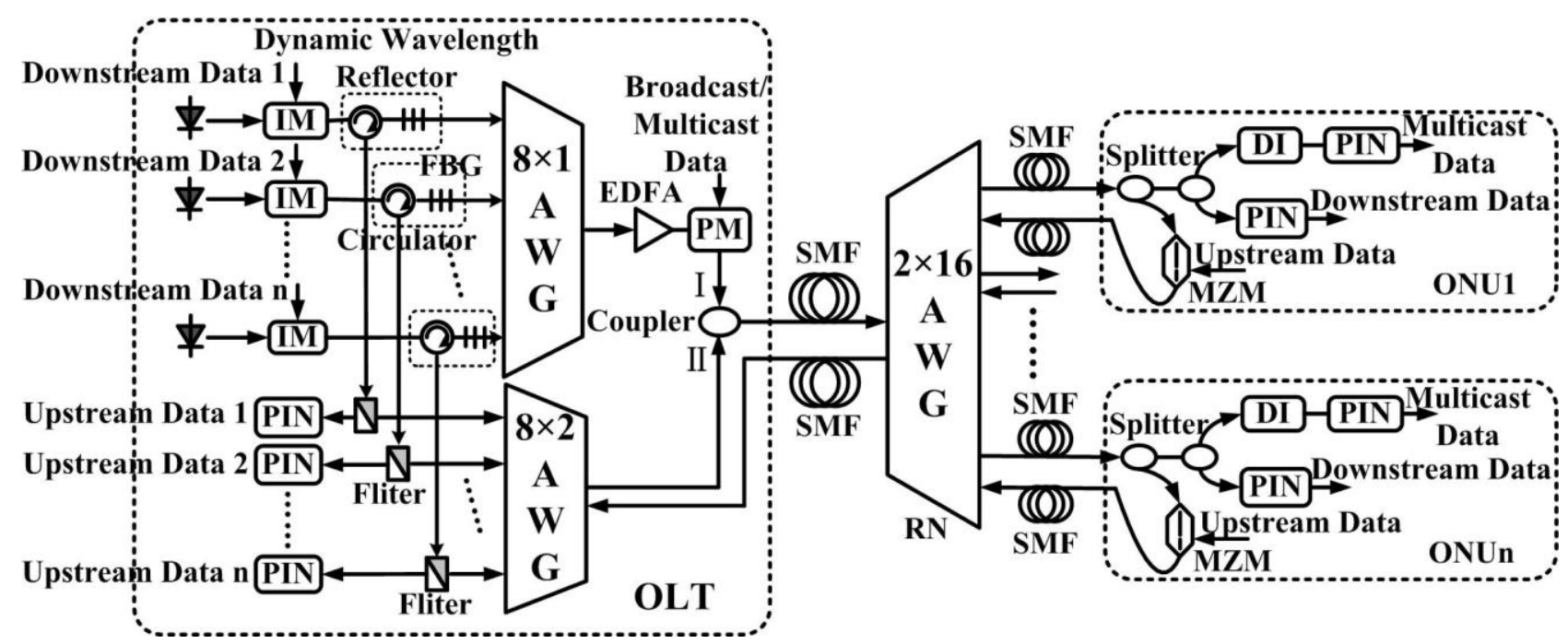

Fig. 1 Schematic diagram of the proposed WDM-PON architecture with multicast overlay

After transmission over fiber, a $2 \times 16$ AWG is used to de-multiplex the optical signals and route them to individual ONUs. In ONU, one part of downstream power is tapped off by an optical splitter for NRZ downstream unicast data and differential-phase-shift-keying (DPSK) multicast data, respectively. For users who subscribe to multicast service, the optical carrier not only carries the downstream unicast data, but also contains the multicast data information. The remaining part is re-modulated by upstream data with higher ER and sent back to the OLT. In this way, colorless ONU without the need of light source could also be implemented. It is an attractive feature for PON deployment.

\section{EXPERIMENTAL SETUP AND RESULTS}

To verify the feasibility of the proposed scheme, we demonstrate downstream unicast/multicast transmission in two different wavelength channels and upstream data re-modulation in Fig.2.

In the OLT, continues wave $(\mathrm{CW})$ in the $1551.95 \mathrm{~nm}$ wavelength is fed into a Mach-Zehnder modulator (MZM) which is biased at the quadrature point and is driven by a $5-\mathrm{Gb} / \mathrm{s} 2^{31}-1$ pseudorandom binary sequence (PRBS) to generate downstream unicast NRZ signal, with $3 \mathrm{~dB}$ ER. After an EDFA and a tunable band-pass filter (BPF) with a bandwidth of $0.4 \mathrm{~nm}$, the unicast NRZ signal is orthogonally phase-modulated by a PM to superimpose multicast data. To obtain better 
signal performance, downstream unicast data and multicast data signals need to be bit synchronized, which is adjusted by an electrical phase shifter.

Another CW at $1548.84 \mathrm{~nm}$ is just modulated by unicast data with 5-Gb/s complementary PRBS and then is bypassed by an optical circulator and FBG with a $3-\mathrm{dB}$ bandwidth of $0.15 \mathrm{~nm}$ and a $90 \%$ reflection ratio. Thus, the downstream optical carrier just contains unicast data. Then, both of the above wavelengths are combined and fed into two pieces of $12.5 \mathrm{~km}$ single mode fiber (SMF) to reach respective ONU. An AWG located in the Remote Node (RN) links the two pieces of SMF.

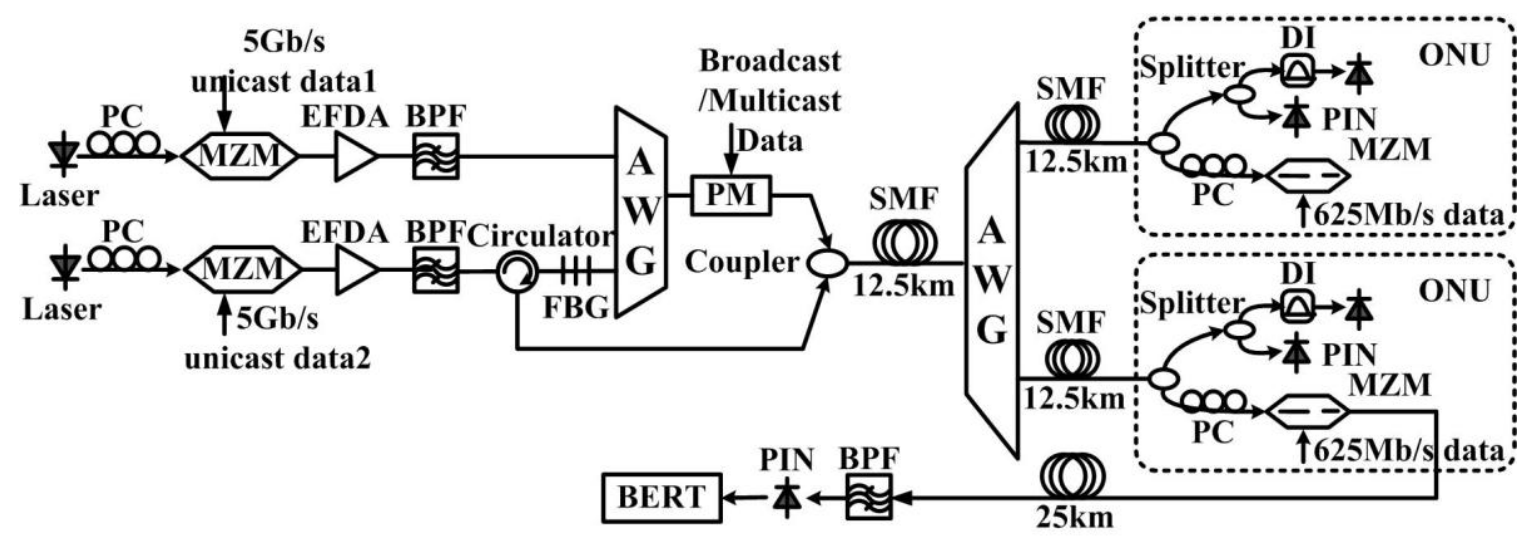

Fig. 2 Experimental setup of the proposed scheme

After transmission over 25-km SMF, at the ONU, an optical splitter is used to tap optical carrier power into two optical paths. The part of the received downstream signal power is again split into two parts. One half is detected for NRZ downstream unicast data and another half is demodulated by a delay interferometer (DI) for DPSK multicast data. The remaining part is re-modulated by $625-\mathrm{Mb} / \mathrm{s}$ upstream data with a higher ER of $\sim 10 \mathrm{~dB}$ and sent back to the OLT.

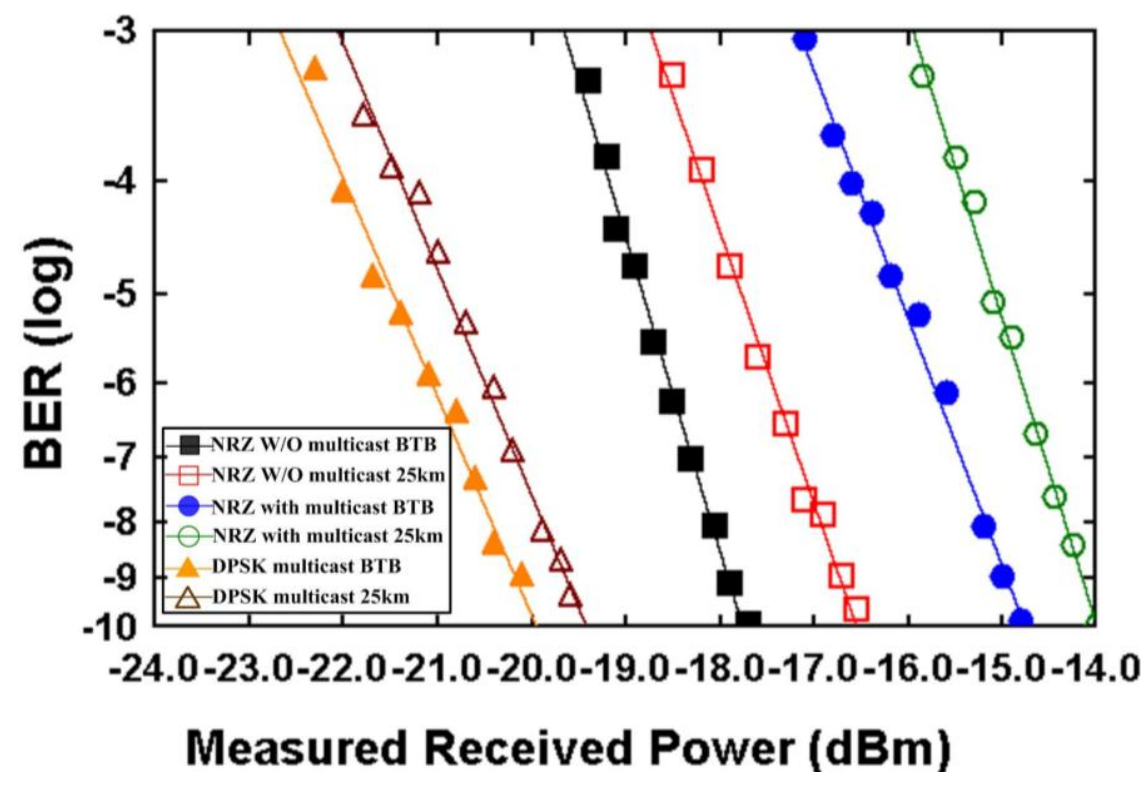

Fig. 3 (a) BER curves for the downstream NRZ/DPSK 
The bit-error-rate (BER) curves and eye diagrams of the downstream unicast NRZ signal and multicast DPSK signal are provided in Fig. 3 a-b). The power penalties of downstream unicast signal caused by the fiber transmission are $\sim 0.8 \mathrm{~dB}$ with multicast data and $\sim 1.3 \mathrm{~dB}$ without multicast data, respectively. The power penalty is $\sim 0.5 \mathrm{~dB}$ for the DPSK multicast signal.

The four eye diagrams are observed in Fig. 3 b). The inset figure 1) shows the eye diagram of the demodulated multicast DPSK signal with downstream unicast NRZ, which has three folds of up-eyelids. The inset figures 2) and 3) display the eye diagrams for the demodulated NRZ with DPSK signal and the demodulated NRZ without DPSK signal, respectively. We observe that the performance degradation of the above eye diagrams induced by the superimposition of multicast DPSK signal is represented by the thicker eyelid. The eye diagram for the downstream unicast NRZ demodulated by DI without DPSK is shown in the inset figure 4).
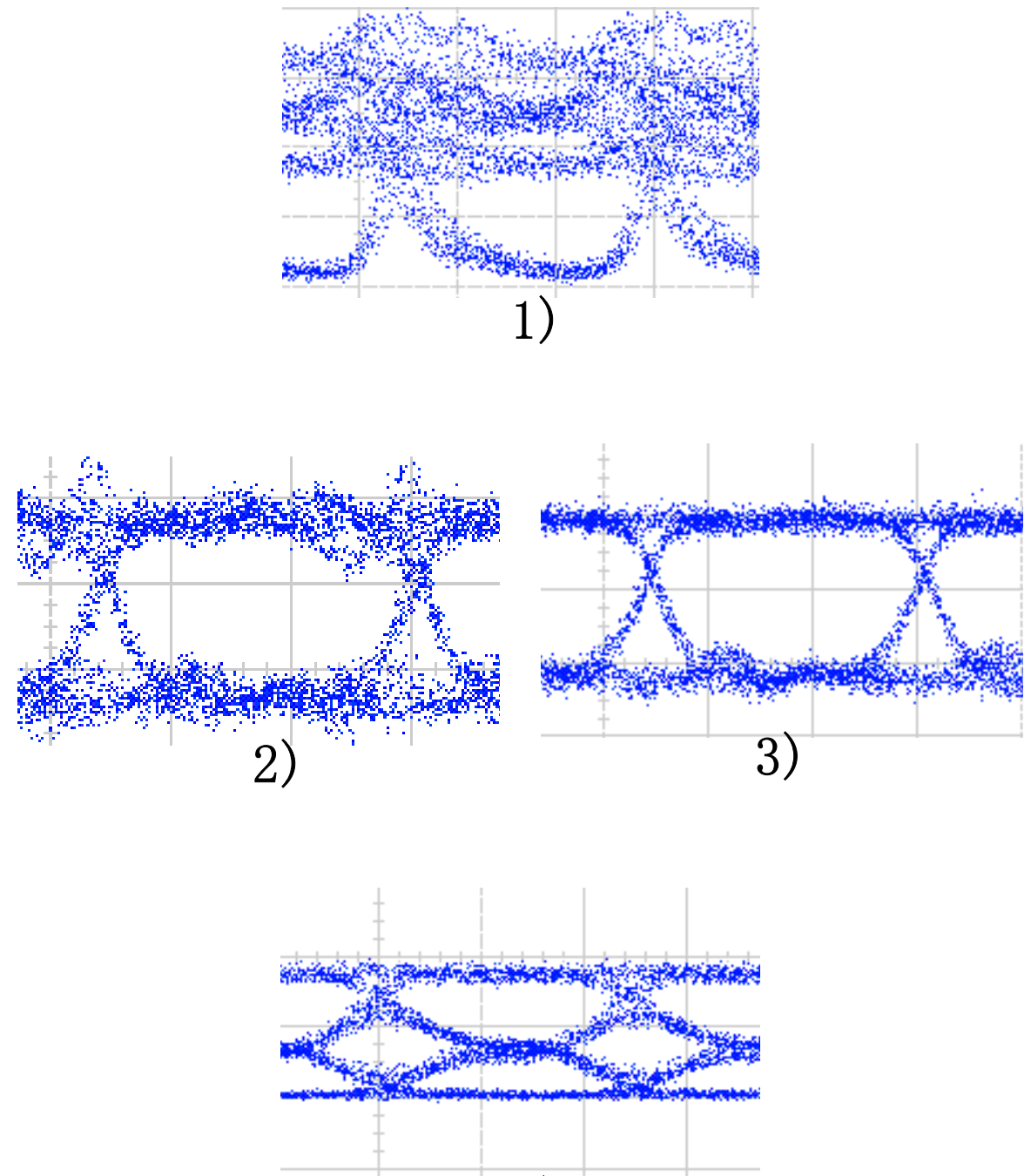

4)

Fig. 3 (b) Eye diagrams of 1) the downstream demodulated DPSK with NRZ, 2) the demodulated NRZ with DPSK, 3) the NRZ demodulated by DI without DPSK, 4) the demodulated NRZ without DPSK 
The Fig. 3 c) shows the BER measurements of upstream re-modulated NRZ signal with higher ER, after the 25-km SMF transmission. We observe that the upstream transmission suffers $\sim 0.5-\mathrm{dB}$ penalty due to the dispersion. The insets of Fig. 3 c) are eye diagrams of upstream re-modulated NRZ signal.

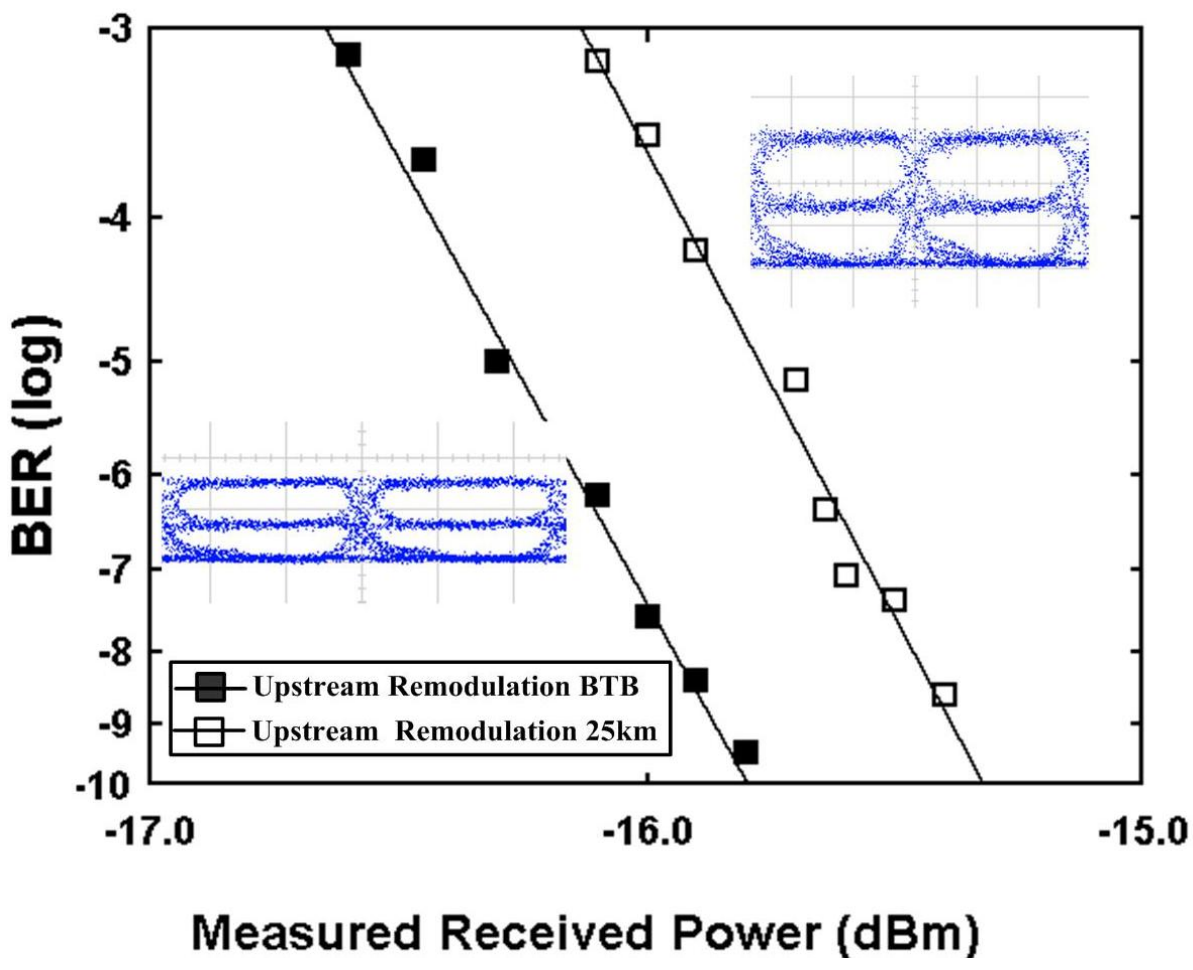

Fig.3 (c) BER curves and eye diagrams for upstream re-modulated signal.

\section{CONCLUSIONS}

We have proposed and experimentally demonstrated a novel scheme to simultaneously deliver downstream unicast and multicast signal based on dynamic wavelength reflection scheme over WDM-PON architecture. In each WDM channel, a dynamic wavelength reflector is used to selectively bypass the corresponding optical carrier to avoid multicast data superimposition, while the remaining optical carriers go through the dynamic wavelength reflector and simultaneously carry the multicast data. The multicast function can be quickly and dynamically reconfigured with simple and centralized control in the OLT and is transparent for all ONUs. The technique just adds a little complexity to the existing WDMPON structure, and its feasibility is demonstrated with $5-\mathrm{Gb} / \mathrm{s}$ downstream unicast, multicast signal and $625-\mathrm{Mb} / \mathrm{s}$ upstream re-modulated data. By employing re-modulation technique, no light sources and colored components are needed in the ONUs, which effectively reduce the cost and complexity.

Acknowledgements: The work was jointly supported by the National Nature Science Fund of China (No. 60972032 and No.60632010) and the National "863" Hi-tech Project of China (No. 2006AA01Z251 and No. 2007AA01Z271). 


\section{REFERENCES}

1. N. Deng, “A WDM Passive Optical Network with Centralized Light Sources and Multicast Overlay,” IEEE PTL, 20, 114-116, 2008.

2. L. Cai, "Video-Service-Overlaid Wavelength-Division-Multiplexed Passive Optical Network," IEEE PTL, 21, 990992, 2009.

3. M. Khanal,, "Selective multicasting of Digital Video Signals over a WDM Passive Optical Network," IEEE PTL, 17, 1992-1994, 2005.

4. J-H. Moon, "Overlay of multicasting Signal in a WDM-PON", OFC/NFOEC 2006, paper OThK8.

5. M. Ohm, “Quaternary Optical ASK-DPSK and Receivers with Direct Detection,” IEEE PTL, 15, 159-161, 2003. 